International Journal of Trend in Scientific Research and Development(IJTSRD)

ISSN:2456-6470 —www.ijtsrd.com-Volume -2-Issue-3

\title{
CRACK PROBLEMS CONCERNING BOUNDARIES OF CONVEX LENS LIKE FORMS
}

\author{
DOO-SUNG LEE \\ Department of Mathematics \\ College of Education, Konkuk University \\ 120 Neungdong-Ro, Kwangjin-Gu, Seoul, Korea \\ e-mail address: dslee@kumail.konkuk.ac.kr
}

\begin{abstract}
The singular stress problem of a peripheral edge crack around a cavity of spherical portion in an infinite elastic medium when the crack is subjected to a known pressure is investigated. The problem is solved by using integral transforms and is reduced to the solution of a singular integral equation of the first kind. The solution of this equation is obtained numerically by the method due to Erdogan, Gupta, and Cook, and the stress intensity factors are displayed graphically.
\end{abstract}

Also investigated in this paper is the pennyshaped crack situated symmetrically on the central plane of a convex lens shaped elastic material.

Key words: cavity of spherical portion/ peripheral edge crack/penny-shaped crack /SIF.

\section{Introduction.}

The problem of determining the distribution of stress in an elastic medium containing a circumferential edge crack has been investigated by several researchers including the present author. Among these investigations, the notable ones are Keer et al.[1,2],Atsumi and Shindo[3,4], and Lee[5,6]. Keer et al.[1] considered a circumferential edge crack in an extended elastic medium with a cylindrical cavity the analysis of which provides immediate application to the study of cracking of pipes and nozzles if the crack is small.
Another important problem involving a circumferential edge crack is that concerned with a spherical cavity. Atsumi and Shindo[4] investigated the singular stress problem of a peripheral edge crack around a spherical cavity under uniaxial tension field. In more recent years, Wan et al.[7] obtained the solution for cracks emanating from surface semi-spherical cavity in finite body using energy release rate theory. In previous studies concerning the spherical cavity with the circumferential edge crack, the cavity was a full spherical shape. In this present analysis, we are concerned with a cavity of a spherical portion, rather than a full spherical cavity. More briefly describing it, the cavity looks like a convex lens. Here, we employ the known methods of previous investigators to derive a singular integral equation of the first kind which was solved numerically, and obtained the s.i.f. for various spherical portions. It is also shown that when this spherical portion becomes a full sphere, the present solution completely agrees with the already known solution.

\section{Formulation of problem and reduction to singular integral equation. ${ }^{0}$}

We employ cylindrical coordinates $(r, \phi, z)$ with the plane $z=0$ coinciding the plane of peripheral edge crack. The spherical coordinates

\footnotetext{
0@IJTSRD— Available Online@www.ijtsrd.com-Volume-2-Issue-3-Mar-Apr 2018 Page:982
} 
$(\rho, \theta, \phi)$ are connected with the cylindrical coordinates by

$$
z=\rho \cos \theta, \quad r=\rho \sin \theta .
$$

Spherical coordinates $(\zeta, \vartheta, \phi)$ whose origin is located at $z=-\delta, \quad r=0$, and is the center of the upper spherical surface, are also used. The cavity is symmetrical with respect to the plane $z=0$

The crack occupies the region $z=0,1 \leq$ $r \leq \gamma$. So the radius of the spherical cavity is $\zeta_{0}=\sqrt{1+\delta^{2}}$.

The boundary conditions are:

On the plane $z=0$, we want the continuity of the shear stress, and the normal displacement:

$$
\begin{gathered}
u_{z}\left(r, 0^{+}\right)-u_{z}\left(r, 0^{-}\right)=0, \quad \gamma \leq r<\infty, \\
\sigma_{r z}\left(r, 0^{+}\right)-\sigma_{r z}\left(r, 0^{-}\right)=0, \quad 1 \leq r<\infty .
\end{gathered}
$$

And the crack is subjected to a known pressure $p(r)$, i.e.,

$$
\sigma_{z z}\left(r, 0^{+}\right)=-p(r), \quad 1 \leq r \leq \gamma .
$$

On the surface of the spherical cavity, stresses are zero:

$$
\begin{aligned}
& \sigma_{\zeta \zeta}\left(\zeta_{0}, \vartheta\right)=0 \\
& \sigma_{\zeta \vartheta}\left(\zeta_{0}, \vartheta\right)=0 .
\end{aligned}
$$

We can make use of the axially symmetric solution of the equations of elastic equilibrium due to Green and Zerna [8] which states that if $\varphi(r, z)$ and $\psi(r, z)$ are axisymmetric solutions of Laplace equation, then the equations

$$
\begin{gathered}
2 \mu u_{r}=\frac{\partial \varphi}{\partial r}+z \frac{\partial \psi}{\partial r}, \\
2 \mu u_{z}=\frac{\partial \varphi}{\partial z}+z \frac{\partial \psi}{\partial z}-(3-4 \nu) \psi,
\end{gathered}
$$

where $\mu$ is the modulus of rigidity and $\nu$ is Poisson's ratio, provide a possible displacement field. The needed components of stress tensor are given by the equations

$$
\begin{gathered}
\sigma_{r z}=\frac{\partial^{2} \phi}{\partial r \partial z}+z \frac{\partial^{2} \psi}{\partial r \partial z}-(1-2 \nu) \frac{\partial \psi}{\partial r}, \\
\sigma_{z z}=\frac{\partial^{2} \phi}{\partial z^{2}}+z \frac{\partial^{2} \psi}{\partial z^{2}}-2(1-\nu) \frac{\partial \psi}{\partial z} .
\end{gathered}
$$

The functions $\phi^{(1)}$ and $\phi^{(2)}$ for the regions $z>0$ and $z<0$, respectively, are chosen as follows:

$$
\begin{aligned}
\phi^{(1)}(r, z)= & (2 \nu-1) \int_{0}^{\infty} \xi^{-1} A(\xi) J_{0}(\xi r) e^{-\xi z} d \xi \\
& +\sum_{n=0}^{\infty} a_{n} \frac{P_{n}(\cos \theta)}{\rho^{n+1}}, \\
\phi^{(2)}(r, z)= & (2 \nu-1) \int_{0}^{\infty} \xi^{-1} A(\xi) J_{0}(\xi r) e^{\xi z} d \xi \\
& -\sum_{n=0}^{\infty} a_{n}(-1)^{n} \frac{P_{n}(\cos \theta)}{\rho^{n+1}} .
\end{aligned}
$$

Here the superscripts (1) and (2) are taken for the region $z>0$ and $z<0$, respectively. The functions $\psi^{(1)}$ and $\psi^{(2)}$ are chosen as follows:

$$
\begin{aligned}
\psi^{(1)}(r, z) & =\int_{0}^{\infty} A(\xi) J_{0}(\xi r) e^{-\xi z} d \xi \\
+ & \sum_{n=0}^{\infty} b_{n} \frac{P_{n}(\cos \theta)}{\rho^{n+1}}, \\
\psi^{(2)}(r, z) & =-\int_{0}^{\infty} A(\xi) J_{0}(\xi r) e^{\xi z} d \xi \\
+ & \sum_{n=0}^{\infty} b_{n}(-1)^{n} \frac{P_{n}(\cos \theta)}{\rho^{n+1}} .
\end{aligned}
$$

Then we can immediately satisfy condition $(2.2)$ by this choice of functions (2.10)-(2.13).

Now the condition (2.1) requires

$$
\int_{0}^{\infty} A(\xi) J_{0}(\xi r) d \xi=0, \quad r>\gamma
$$

0

Equation (2.14) is automatically satisfied by setting

$$
A(\xi)=\int_{1}^{\gamma} t g(t) J_{1}(\xi t) d t
$$

\footnotetext{
0@IJTSRD— Available Online@www.ijtsrd.com-Volume-2-Issue-3-Mar-Apr 2018 Page:983
} 
Then from the boundary condition (2.3), we obtain

$$
\begin{gathered}
\int_{0}^{\infty} \xi A(\xi) J_{0}(\xi r) d \xi-\sum_{n=0}^{\infty} a_{2 n} \frac{(2 n+1)^{2} P_{2 n}(0)}{r^{2 n+3}} \\
-2(1-\nu) \sum_{n=0}^{\infty} b_{2 n+1} \frac{P_{2 n+1}^{\prime}(0)}{r^{2 n+3}}=-p(r) \\
1 \leq r \leq \gamma
\end{gathered}
$$

where prime indicates the differentiation with respect to the argument.

By substituting (2.15) into (2.16), it reduces to

$$
\begin{gathered}
-\frac{2}{\pi} \int_{1}^{\gamma} t g(t) R(r, t) d t-\sum_{n=0}^{\infty} r^{-(2 n+3)}\left\{P_{2 n}(0)\right. \\
\left.\times(2 n+1)^{2} a_{2 n}+\alpha b_{2 n+1} P_{2 n+1}^{\prime}(0)\right\}=-p(r), \\
1 \leq r \leq \gamma,
\end{gathered}
$$

where

$$
\begin{aligned}
R(r, t) & =\frac{1}{r^{2}-t^{2}} E\left(\frac{r}{t}\right), \quad t>r, \\
& =\frac{r}{t} \frac{1}{r^{2}-t^{2}} E\left(\frac{t}{r}\right)-\frac{1}{r t} K\left(\frac{t}{r}\right), \quad r>t .
\end{aligned}
$$

$K$ and $E$ in (2.18) are complete elliptic integrals of the first and the second kind, respectively and $\alpha=2(1-\nu)$.

The solution will be complete, if the conditions on the surface of the spherical cavity are satisfied.

\section{Conditions on the surface of the spheri- cal cavity.}

Equation (2.17) gives one relation connecting unknown coefficients $a_{n}$ and $b_{n}$. The stress components besides (2.8) and (2.9) which are needed for the present analysis are given by the following equations

$$
\begin{gathered}
\sigma_{\zeta \zeta}=\frac{\partial^{2} \phi}{\partial \zeta^{2}}+\zeta \\
\cos \vartheta \frac{\partial^{2} \psi}{\partial \zeta^{2}}-2(1-\nu) \cos \vartheta \frac{\partial \psi}{\partial \zeta} \\
+2 \nu \frac{\sin \vartheta}{\zeta} \frac{\partial \psi}{\partial \vartheta}
\end{gathered}
$$

$$
\begin{array}{r}
\sigma_{\zeta \vartheta}=\frac{1}{\zeta} \frac{\partial^{2} \phi}{\partial \zeta \partial \vartheta}-\frac{1}{\zeta^{2}} \frac{\partial \phi}{\partial \vartheta}+\cos \vartheta \frac{\partial^{2} \psi}{\partial \zeta \partial \vartheta} \\
+(1-2 \nu) \sin \vartheta \frac{\partial \psi}{\partial \zeta}-2(1-\nu) \frac{\cos \vartheta}{\zeta} \frac{\partial \psi}{\partial \vartheta} .
\end{array}
$$

To satisfy boundary conditions on the spherical surface, it is needed to represent $\phi, \psi$ in (2.10)(2.13) in terms of $\zeta, \vartheta$ variables. To do so we utilize the following formula whose validity is shown in the Appendix 1. An expression useful for the present analysis is the following

$$
\frac{P_{n}(\cos \theta)}{\rho^{n+1}}=\sum_{k=0}^{\infty}\left(\begin{array}{c}
n+k \\
k
\end{array}\right) \frac{P_{n+k}(\cos \vartheta)}{\zeta^{n+k+1}} \delta^{k} .
$$

Thus

$$
\begin{aligned}
& \sum_{n=0}^{\infty} a_{n} \frac{P_{n}(\cos \theta)}{\rho^{n+1}}=\sum_{n=0}^{\infty} a_{n} \sum_{k=0}^{\infty}\left(\begin{array}{c}
n+k \\
k
\end{array}\right) \\
& \times \frac{P_{n+k}(\cos \vartheta)}{\zeta^{n+k+1}} \delta^{k}=\sum_{j=0}^{\infty} \frac{P_{j}(\cos \vartheta)}{\zeta^{j+1}} A_{j}
\end{aligned}
$$

where

$$
A_{j}=\sum_{n=0}^{j} \frac{j !}{(j-n) ! n !} a_{n} \delta^{j-n} .
$$

Also

$$
\begin{aligned}
& \int_{0}^{\infty} \xi^{-1} A(\xi) J_{0}(\xi r) e^{-\xi z} d \xi=\int_{1}^{\gamma} t g(t) \\
& \times \int_{0}^{\infty} \xi^{-1} J_{0}(\xi r) J_{1}(\xi t) e^{-\xi z} d \xi d t .
\end{aligned}
$$

If we make use of the formula in Whittaker and Watson[9,pp.395-396]

$\int_{-\pi}^{\pi} \exp \{-\xi(z+i x \cos u+i y \sin u)\} d u=2 \pi e^{-\xi z} J_{0}(\xi r)$,

${ }^{0}$ to the inner integral of (3.6), it can be written as, if we are using the shortened notation

$$
\beta=z+i x \cos u+i y \sin u,
$$

then

$$
\begin{aligned}
& \int_{0}^{\infty} \xi^{-1} J_{0}(\xi r) J_{1}(\xi t) e^{-\xi z} d \xi \\
= & \frac{1}{2 \pi} \int_{-\pi}^{\pi} \int_{0}^{\infty} \xi^{-1} J_{1}(\xi t) e^{-\xi \beta} d \xi d u
\end{aligned}
$$

\footnotetext{
0@IJTSRD—Available Online@www.ijtsrd.com-Volume-2-Issue-3-Mar-Apr 2018 Page:984
} 


$$
\begin{gathered}
=\frac{1}{2 \pi} \int_{-\pi}^{\pi} \frac{t}{\beta+\sqrt{\beta^{2}+t^{2}}} d u \\
=-\frac{1}{2 \pi} \int_{-\pi}^{\pi}\left\{\frac{\beta}{t}-\sqrt{1+\frac{\beta^{2}}{t^{2}}}\right\} d u \\
=-\frac{1}{2 \pi} \int_{-\pi}^{\pi}\left\{\frac{\beta}{t}-\sum_{n=0}^{\infty} \frac{\left(-\frac{1}{2}\right)_{n}(-1)^{n}}{n !}\left(\frac{\beta}{t}\right)^{2 n}\right\} d u .
\end{gathered}
$$

As $|\beta|<|t|$, the above series expansion is valid. Next,

$$
\begin{aligned}
\beta^{2 n}=(-\delta & +Z+i x \cos u+i y \sin u)^{2 n}=\left(-\delta+\beta^{\prime}\right)^{2 n} \\
= & \sum_{k=0}^{2 n}\left(\begin{array}{c}
2 n \\
k
\end{array}\right)(-\delta)^{2 n-k}\left(\beta^{\prime}\right)^{k},
\end{aligned}
$$

where $(r, \phi, Z)$ is the cylindrical coordinates system centered at $(r, z)=(0,-\delta)$. We have the following formula from Whittaker and Watson $[9$, p.392]

$$
\begin{aligned}
\int_{-\pi}^{\pi}\left(\beta^{\prime}\right)^{n} d u= & \int_{-\pi}^{\pi}(Z+i x \cos u+i y \sin u)^{n} d u \\
& =2 \pi \zeta^{n} P_{n}(\cos \vartheta) .
\end{aligned}
$$

If we utilize (3.8) and (3.9) in (3.7), it can be written as

$$
\begin{gathered}
\int_{0}^{\infty} \xi^{-1} J_{0}(\xi r) J_{1}(\xi t) e^{-\xi z} d \xi \\
=\sum_{n=0}^{\infty} \frac{\left(-\frac{1}{2}\right)_{n}(-1)^{n}}{n !} \frac{1}{t^{2 n}} \\
\times \sum_{k=0}^{2 n}\left(\begin{array}{c}
2 n \\
k
\end{array}\right)(-\delta)^{2 n-k} \zeta^{k} P_{n}(\cos \vartheta) \\
+\delta-\frac{\zeta}{t} P_{1}(\cos \vartheta) .
\end{gathered}
$$

Thus finally, from (3.4), (3.6) and (3.10), $\phi^{(1)}$ can be written in terms of spherical coordinates $(\zeta, \vartheta)$ as

$$
\begin{gathered}
\phi^{(1)}=\sum_{k=0}^{\infty} P_{k}(\cos \vartheta)\left[\frac{A_{k}}{\zeta^{k+1}}+\Phi_{k} \zeta^{k}\right] \\
+\delta \int_{1}^{\gamma} t g(t) d t-\zeta P_{1}(\cos \vartheta) \int_{1}^{\gamma} g(t) d t,
\end{gathered}
$$

where

$$
\Phi_{k}=-(\alpha-1) \sum_{n=[(k+1) / 2]}^{\infty} \frac{\left(-\frac{1}{2}\right)_{n}(-1)^{n}}{n !}
$$

$$
\times \frac{(2 n) !}{(2 n-k) ! k !}(-\delta)^{2 n-k} \int_{1}^{\gamma} \frac{g(t)}{t^{2 n-1}} d t,
$$

and $[(\mathrm{k}+1) / 2]$ is the greatest integer $\leq(k+1) / 2$.

It is also necessary to express $\psi^{(1)}$ in terms of spherical coordinates $(\zeta, \vartheta)$. Now, as in (3.4)

$$
\sum_{n=0}^{\infty} b_{n} \frac{P_{n}(\cos \theta)}{\rho^{n+1}}=\sum_{j=0}^{\infty} \frac{P_{j}(\cos \vartheta)}{\zeta^{j+1}} B_{j},
$$

where

$$
B_{j}=\sum_{n=0}^{\infty} \frac{j !}{(j-n) ! n !} b_{n} \delta^{j-n}
$$

We first express following integral in $(\rho, \theta)$ coordinates

$$
\begin{aligned}
& \int_{0}^{\infty} A(\xi) J_{0}(\xi r) e^{-\xi z} d \xi=\int_{1}^{\gamma} t g(t) d t \\
& \quad \times \int_{0}^{\infty} J_{1}(\xi t) J_{0}(\xi r) e^{-\xi z} d \xi
\end{aligned}
$$

The inner integral on the right-hand side of (3.13) is

$$
\begin{gathered}
-\frac{1}{t} \int_{0}^{\infty} J_{0}(\xi r) e^{-\xi z} \frac{\partial}{\partial \xi} J_{0}(\xi t) d \xi=\frac{1}{t} \\
-\frac{1}{t} \int_{0}^{\infty}\left\{r J_{1}(\xi r)+z J_{0}(\xi r)\right\} e^{-\xi z} J_{0}(\xi t) d \xi .
\end{gathered}
$$

Using the equation in Erdélyi et al.[10] ${ }^{0}$

$$
J_{0}(\xi t)=\frac{2}{\pi} \int_{t}^{\infty} \frac{\sin (\xi x) d x}{\sqrt{x^{2}-t^{2}}}
$$

equation (3.14) is equal to

$$
\frac{1}{t}+\frac{1}{t} \frac{2}{\pi} \int_{t}^{\infty} \frac{d x}{\sqrt{x^{2}-t^{2}}}
$$

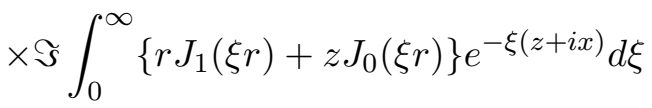

$$
=\frac{1}{t}+\frac{1}{t} \frac{2}{\pi} \int_{t}^{\infty} \frac{1}{\sqrt{x^{2}-t^{2}}} \Im \frac{-i x}{\sqrt{r^{2}+(z+i x)^{2}}} d x
$$$$
=\frac{1}{t}-\frac{1}{t} \frac{2}{\pi} \int_{t}^{\infty} \frac{1}{\sqrt{x^{2}-t^{2}}} \Im \frac{d x}{\sqrt{1-2 \frac{\rho}{x} i \cos \theta+\left(\frac{\rho}{x} i\right)^{2}}}
$$$$
=\frac{1}{t}-\frac{1}{t} \sum_{n=0}^{\infty}\left(\frac{\rho}{t}\right)^{2 n+1}(-1)^{n} P_{2 n+1}(\cos \theta) \frac{\left(\frac{1}{2}\right)_{n}}{n !},
$$

\footnotetext{
0@IJTSRD—Available Online@www.ijtsrd.com-Volume-2-Issue-3-Mar-Apr 2018 Page:985
} 
INTERNATIONAL JOURNAL OF TREND IN SCIENTIFIC RESEARCH AND DEVELOPMENT(IJTSRD)ISSN:2456-6470

where we used the generating function

$$
\frac{1}{\sqrt{1-2 x \cos \theta+x^{2}}}=\sum_{n=0}^{\infty} P_{n}(\cos \theta) x^{n} .
$$

To express $(3.15)$ in the $(\zeta, \vartheta)$ spherical coordinates, we use the following formula whose validity is shown in the Appendix 2.

$$
\begin{aligned}
\rho^{2 n+1} & P_{2 n+1}(\cos \theta)=\sum_{k=0}^{2 n+1} \frac{(2 n+1) !}{k !(2 n+1-k) !} \\
& \times(-\delta)^{2 n+1-k} \zeta^{k} P_{k}(\cos \vartheta) .
\end{aligned}
$$

${ }^{0}$ If we use (3.16) in (3.15), then with (3.12), we get $\psi^{(1)}$ in spherical coordinates $(\zeta, \vartheta)$

$\psi^{(1)}=\sum_{k=0}^{\infty} P_{k}(\cos \vartheta)\left(\frac{B_{k}}{\zeta^{k+1}}+\Psi_{k} \zeta^{k}\right)+\int_{1}^{\gamma} g(t) d t$,

where

$$
\begin{aligned}
\Psi_{k}=- & \sum_{n=[k / 2]}^{\infty} \frac{(-1)^{n}\left(\frac{1}{2}\right)_{n}}{n !} \frac{(2 n+1) !}{k !(2 n+1-k) !} \\
& \times(-\delta)^{2 n+1-k} \int_{1}^{\gamma} \frac{g(t)}{t^{2 n+1}} d t .
\end{aligned}
$$

Then if we substitute these values of $\phi^{(1)}$ and $\psi^{(1)}$ given by (3.11) and (3.17) into (3.2), and simplify the results by using the properties of Legendre polynomials, from the condition that on the spherical surface $\zeta=\zeta_{0}$, the shear stress $\sigma_{\zeta \vartheta}=0$, we obtain the following equation

$$
\begin{gathered}
-A_{k+1} \frac{k+3}{\zeta_{0}^{k+4}}+\frac{B_{k}}{\zeta_{0}^{k+2}} \frac{\alpha-(k+1)^{2}}{2 k+1} \\
-\frac{B_{k+2}}{\zeta_{0}^{k+4}} \frac{(k+3)(2 \alpha+k+2)}{2 k+5} \\
+k \zeta_{0}^{k-1} \Phi_{k+1}+\zeta_{0}^{k-1} \Psi_{k} \frac{k(k+1-2 \alpha)}{2 k+1} \\
-\zeta_{0}^{k+1} \Psi_{k+2} \frac{k+2+\alpha-(k+2)(k+3)}{2 k+5}=0 .
\end{gathered}
$$

Likewise, from the condition $\sigma_{\zeta \zeta}\left(\zeta_{0}, \vartheta\right)=0$, we get following two equations,

$$
-A_{0} \frac{2}{\zeta_{0}^{3}}-\frac{B_{1}}{\zeta_{0}^{3}} \frac{2(2 \alpha+1)}{3}-\frac{1}{3} \Psi_{1}(\alpha-4)=0
$$

$$
\begin{gathered}
-A_{k+1} \frac{(k+2)(k+3)}{\zeta_{0}^{k+4}} \\
+\frac{B_{k}}{\zeta_{0}^{k+2}} \frac{(k+1)\{2-\alpha-(k+1)(k+4)\}}{2 k+1} \\
-\frac{B_{k+2}}{\zeta_{0}^{k+4}} \frac{(k+2)(k+3)(2 \alpha+k+2)}{2 k+5} \\
-k(k+1) \zeta_{0}^{k-1} \Phi_{k+1}-\zeta_{0}^{k-1} \Psi_{k} \frac{k(k+1)(k+1-2 \alpha)}{2 k+1} \\
-\zeta_{0}^{k+1} \Psi_{k+2} \frac{(k+2)\{(k+2)(k-1)+\alpha-2\}}{2 k+5}=0 .
\end{gathered}
$$

Thus if we multiply (3.18) by $k+2$ and subtract $(3.19 \mathrm{~b})$ from the resulting equation we find

$$
\begin{aligned}
& B_{k}=-\frac{\zeta_{0}^{k+2}(2 k+1)}{\alpha(2 k+3)+2 k(k+1)}\left[k(2 k+3) \zeta_{0}^{k-1} \Phi_{k+1}\right. \\
& \left.+\zeta_{0}^{k-1} \Psi_{k} \frac{(2 k+3) k(k+1-2 \alpha)}{2 k+1}+\zeta_{0}^{k+1} \Psi_{k+2} k(k+2)\right]
\end{aligned}
$$

If we solve (3.20) for $b_{i}$ using the theorem which is in the Appendix 3 and the relation,

$$
\Phi_{k+1}=\frac{\alpha-1}{k+1} \Psi_{k},
$$

we find

$$
\frac{b_{i}}{\delta^{i}}=\sum_{k=0}^{i} \frac{i !(-1)^{i-k}}{(i-k) ! k !} \frac{1}{\delta^{k}}\left[N_{1}(k) \Psi_{k}+N_{2}(k) \Psi_{k+2}\right],
$$

where

$$
\begin{gathered}
N_{1}(k)=-\frac{\zeta_{0}^{2 k+1}(2 k+3) k}{\alpha(2 k+3)+2 k(k+1)}\left(\frac{-\alpha+k^{2}}{k+1}\right), \\
N_{2}(k)=-\frac{\zeta_{0}^{2 k+3}(2 k+1) k(k+2)}{\alpha(2 k+3)+2 k(k+1)} .
\end{gathered}
$$

Equation (3.21) can be written as

$$
\begin{gathered}
b_{i}=-\sum_{k=0}^{i} \frac{i !(-1)^{i-k}}{(i-k) ! k !} \frac{\delta^{i}}{\delta^{k}} \int_{1}^{\gamma} \frac{g(t)}{t} \\
\times \sum_{n=0}^{1}\left[N_{n+1}(k) f_{k+2 n}\left(\frac{1}{2}, \frac{\delta}{t}\right) d t,\right.
\end{gathered}
$$

\footnotetext{
0@IJTSRD—Available Online@www.ijtsrd.com-Volume-2-Issue-3-Mar-Apr 2018 Page:986
} 
6NTERNATIONAL JOURNAL OF TREND IN SCIENTIFIC RESEARCH AND DEVELOPMENT(IJTSRD)ISSN:2456-6470

where

$f_{k}(c, x)=\frac{(-\delta)^{1-k}}{k !} \sum_{\ell=[k / 2]}^{\infty} \frac{(-1)^{\ell}(c)_{\ell}(2 \ell+1) ! x^{2 \ell}}{(2 \ell+1-k) ! \ell !}$ with $(c)_{\ell}=c(c+1)(c+2) \cdots(c+\ell-1)$.

Now from $(3.19 \mathrm{a}, \mathrm{b})$ we have

$A_{k+1}=M_{1}(k) \Psi_{k}+M_{2}(k) \Psi_{k+2}+M_{3}(k) \Psi_{k+4}$,

where ${ }^{0}$

$$
A_{0}=\tilde{A}_{1} \Psi_{1}+\tilde{A}_{2} \Psi_{3}
$$

$$
\begin{gathered}
M_{1}(k)=-\frac{\zeta_{0}^{2 k+3} k\left(-\alpha+k^{2}\right)}{(k+2)(k+3)(2 k+1)} \\
\times\left[\frac{(2 k+3) \tilde{g}(k)}{h(k)}+1\right], \\
M_{2}(k)=\frac{\zeta_{0}^{2 k+5}}{k+3}\left[-\frac{\tilde{g}(k)}{h(k)} k(k+1)+\frac{\tilde{g}(k-2)}{2 k+5}\right. \\
\left.+\frac{(k+2)(2 k+7)(2 \alpha+k+2)\left\{-\alpha+(k+2)^{2}\right\}}{(2 k+5) h(k+2)}\right], \\
M_{3}(k)=\frac{\zeta_{0}^{2 k+7}(k+2)(k+4)(2 \alpha+k+2)}{h(k+2)}, \\
\tilde{A}_{1}=-\frac{\zeta_{0}^{3}}{6}\left\{\alpha-4-\frac{5(2 \alpha+1)(-\alpha+1)}{h(1)}\right\}, \\
\tilde{A}_{2}=\frac{3(2 \alpha+1) \zeta_{0}^{5}}{h(1)},
\end{gathered}
$$

with

$$
\begin{aligned}
& \tilde{g}(k)=2-\alpha-(k+1)(k+4), \\
& h(k)=\alpha(2 k+3)+2 k(k+1) .
\end{aligned}
$$

In a similar way $a_{i}^{\prime} s$ can be found from these equations as follows:

$$
\begin{gathered}
a_{2 i}=\left(\tilde{A}_{1} \Psi_{1}+\tilde{A}_{2} \Psi_{3}\right) \delta^{2 i} \\
+\sum_{k=0}^{2 i-1} \frac{(2 i) !(-\delta)^{2 i-k-1}}{(2 i-1-k) !(k+1) !} \tilde{F}(k),
\end{gathered}
$$

where

$$
\begin{gathered}
\tilde{F}(k)=-\int_{1}^{\gamma} \frac{g(t)}{t}\left[M_{1}(k) f_{k}\left(\frac{1}{2}, \frac{\delta}{t}\right)+M_{2}(k)\right. \\
\left.\times f_{k+2}\left(\frac{1}{2}, \frac{\delta}{t}\right)+M_{3}(k) f_{k+4}\left(\frac{1}{2}, \frac{\delta}{t}\right)\right] d t .
\end{gathered}
$$

Thus

$$
\begin{gathered}
\sum_{i=0}^{\infty} a_{2 i} \frac{(2 i+1)^{2} P_{2 i}(0)}{r^{2 i+3}}=\frac{a_{0}}{r^{3}}+\sum_{i=1}^{\infty}\left(\tilde{A}_{1} \Psi_{1}+\tilde{A}_{2} \Psi_{3}\right) \\
\times \delta^{2 i} \frac{(2 i+1)^{2}(-1)^{i}\left(\frac{1}{2}\right)_{i}}{r^{2 i+3} i !} \\
\quad+\sum_{i=1}^{\infty} \frac{(2 i+1)^{2}(-1)^{i}\left(\frac{1}{2}\right)_{i}}{r^{2 i+3} i !} \\
\times \sum_{k=0}^{2 i-1} \frac{(2 i) !(-\delta)^{2 i-k-1}}{(2 i-1-k) !(k+1) !} \tilde{F}(k) .
\end{gathered}
$$

If we interchange the order of summation in the last term of the above equation, and use

$$
\begin{aligned}
& \Psi_{1}=-\int_{1}^{\gamma} \frac{g(t)}{t} f_{1}\left(\frac{1}{2}, \frac{\delta}{t}\right) d t, \\
& \Psi_{3}=-\int_{1}^{\gamma} \frac{g(t)}{t} f_{3}\left(\frac{1}{2}, \frac{\delta}{t}\right) d t,
\end{aligned}
$$

we finally obtain

$$
\sum_{i=0}^{\infty} a_{2 i} \frac{(2 i+1)^{2} P_{2 i}(0)}{r^{2 i+3}}=-\int_{1}^{\gamma} t g(t) T_{1}(r, t) d t
$$

where

$$
\begin{gathered}
T_{1}(r, t)=\frac{1}{r^{3} t^{2}} \\
\times\left[\sum_{k=0}^{\infty} \sum_{m=0}^{2} M_{m+1}(k) f_{k+2 m}\left(\frac{1}{2}, \frac{\delta}{t}\right) h_{k}\left(\frac{\delta}{r}\right)\right. \\
\left.+\left\{\tilde{A}_{1} f_{1}\left(\frac{1}{2}, \frac{\delta}{t}\right)+\tilde{A}_{2} f_{3}\left(\frac{1}{2}, \frac{\delta}{t}\right)\right\}\left\{1+j\left(\frac{\delta}{r}\right)\right\}\right],
\end{gathered}
$$

with

$$
h_{k}\left(\frac{\delta}{r}\right)=\frac{(-\delta)^{-k-1}}{(k+1) !}
$$

$$
\begin{aligned}
& \times \sum_{i=[k / 2+1]}^{\infty} \frac{(2 i+1)^{2}(-1)^{i}\left(\frac{1}{2}\right)_{i}(2 i) !}{(2 i-1-k) ! i !}\left(\frac{\delta}{r}\right)^{2 i}, \\
& j\left(\frac{\delta}{r}\right)=\sum_{i=1}^{\infty} \frac{(2 i+1)^{2}(-1)^{i}\left(\frac{1}{2}\right)_{i}}{i !}\left(\frac{\delta}{r}\right)^{2 i} .
\end{aligned}
$$

Also,

$$
\begin{gathered}
\sum_{i=0}^{\infty} b_{2 i+1} \frac{P_{2 i+1}^{\prime}(0)}{r^{2 i+3}}=-\sum_{i=0}^{\infty} \frac{(-1)^{i}\left(\frac{3}{2}\right)_{i}}{i ! r^{2 i+3}} \\
\times \sum_{k=0}^{2 i+1} \frac{(2 i+1) !(-\delta)^{2 i-k+1}}{(2 i+1-k) ! k !}
\end{gathered}
$$

\footnotetext{
0@IJTSRD—Available Online@www.ijtsrd.com-Volume-2-Issue-3-Mar-Apr 2018 Page:987
} 
INTERNATIONAL JOURNAL OF TREND IN SCIENTIFIC RESEARCH AND DEVELOPMENT(IJTSRD)ISSN:2456-647ø

$$
\times \int_{1}^{\gamma} \frac{g(t)}{t} \sum_{n=0}^{1} N_{n+1}(k) f_{k+2 n}\left(\frac{1}{2}, \frac{\delta}{t}\right) d t .
$$

If we change the order of summation in the above equation, we get

$$
\sum_{i=0}^{\infty} b_{2 i+1} \frac{P_{2 i+1}^{\prime}(0)}{r^{2 i+3}}=-\int_{1}^{\gamma} t g(t) T_{2}(r, t) d t
$$

where

$$
\begin{gathered}
T_{2}(r, t)=\frac{1}{t^{2} r^{3}} \sum_{k=0}^{\infty} \sum_{n=0}^{1} N_{n+1}(k) f_{k}\left(\frac{3}{2}, \frac{\delta}{r}\right) \\
\times f_{k+2 n}\left(\frac{1}{2}, \frac{\delta}{t}\right) .
\end{gathered}
$$

Thus finally the singular integral equation (2.17) becomes

$$
\begin{gathered}
\frac{2}{\pi} \int_{1}^{\gamma} \operatorname{tg}(t)\{R(r, t)+S(r, t)\} d t=p(r) \\
1 \leq r \leq \gamma
\end{gathered}
$$

where

$$
S(r, t)=-\frac{\pi}{2}\left\{T_{1}(r, t)+\alpha T_{2}(r, t)\right\} .
$$

When $\delta=0$, we briefly show that this equation completely agrees with what Atsumi and Shindo obtained. Using

$$
\begin{gathered}
\lim _{\delta \rightarrow 0} h_{2 k-1}\left(\frac{\delta}{r}\right)=\frac{(-1)^{k}\left(\frac{1}{2}\right)_{k}(2 k+1)^{2}}{k !} \frac{1}{r^{2 k}}, \\
\lim _{\delta \rightarrow 0} f_{2 k-1}\left(\frac{1}{2}, \frac{\delta}{t}\right)=\frac{(-1)^{k-1}\left(\frac{1}{2}\right)_{k-1}}{(k-1) !} \frac{1}{t^{2 k-2}}, \\
A=\lim _{\delta \rightarrow 0} \sum_{k=0}^{\infty} \sum_{m=0}^{2} M_{m+1}(k) f_{k+2 m}\left(\frac{1}{2}, \frac{\delta}{t}\right) h_{k}\left(\frac{\delta}{r}\right) \\
=\sum_{k=1}^{\infty} \frac{(-1)^{k}\left(\frac{1}{2}\right)_{k}(2 k+1)^{2}}{k ! r^{2 k}} \\
\left.\times \frac{1}{(2 k+1)(2 k+2)} \frac{(2 k-1}{4 k-1} \frac{(4 k+1) G(k)}{H(k)}+1\right\} \\
\times F(k) \frac{(-1)^{k-1}\left(\frac{1}{2}\right)_{k-1} \frac{1}{t^{2 k-2}}}{(k-1) !} \\
+(2 k+1)\left\{-\frac{G(k)}{H(k)}(2 k-1) 2 k\right. \\
+\frac{(2 k+1)(4 k+5)(2 \alpha+2 k+1)}{H(k+1)(4 k+3)} F(k+1)
\end{gathered}
$$

where ${ }^{0}$

$$
\left.+\frac{G(k-1)}{4 k+3}\right\} \frac{(-1)^{k}\left(\frac{1}{2}\right) k}{k !} \frac{1}{t^{2 k}}
$$

$$
+\frac{(2 k+1)^{2}(2 k+2)(2 k+3)(2 \alpha+2 k+1)}{H(k+1)}
$$

$$
\left.\times \frac{(-1)^{k+1}\left(\frac{1}{2}\right)_{k+1}}{(k+1) !} \frac{1}{t^{2 k+2}}\right] \text {, }
$$

$$
\begin{gathered}
H(k)=\alpha(4 k+1)+4 k(2 k-1), \\
G(k)=2-\alpha-2 k(2 k+3), \\
F(k)=-\alpha+(2 k-1)^{2} . \\
B=\lim _{\delta \rightarrow 0} \sum_{k=0}^{\infty} \sum_{n=0}^{1} N_{n+1}(k) f_{k}\left(\frac{3}{2}, \frac{\delta}{r}\right) f_{k+2 n}\left(\frac{1}{2}, \frac{\delta}{t}\right) \\
=\sum_{k=1}^{\infty} \frac{(-1)^{k}\left(\frac{3}{2}\right)_{k}}{k ! r^{2 k}}\left[-\frac{(2 k+1)(4 k+5)}{2(k+1)}\right. \\
\times \frac{F(k+1)}{H(k+1)} \frac{(-1)^{k}\left(\frac{1}{2}\right)_{k}}{k !} \frac{1}{t^{2 k}}-\frac{(4 k+3)(2 k+1)(2 k+3)}{H(k+1)} \\
\left.\times \frac{(-1)^{k+1}\left(\frac{1}{2}\right)_{k+1}}{(k+1) !} \frac{1}{t^{2 k+2}}\right]+\frac{1}{2 H(1)}\left\{-5 F(1)+\frac{9}{t^{2}}\right\} .
\end{gathered}
$$

Thus

$$
\begin{gathered}
A+\alpha B=\sum_{k=1}^{\infty} \frac{(-1)^{k}\left(\frac{1}{2}\right)_{k}(2 k+1)}{k ! r^{2 k}} \\
\times\left[\frac{(-1)^{k}(2 k-1)(2 k+1)}{H(k) k(k+1)}\{k(k+1) F(k)\right. \\
-\frac{1}{t^{2}} \frac{1}{4(4 k+3)}\{(2 k-1)(4 k+3) 2 k G(k) \\
+(-1)^{k} \frac{F(k+1)(2 k+1)}{H(k+1)(2 k+2)}\left\{(2 k+3)(2 k+2) \frac{1}{t^{2}}\right. \\
\left.\left.+F(k+1) \frac{4 k+5}{4 k+3}\right\} \frac{\left(\frac{1}{2}\right) k}{k !} \frac{1}{t^{2 k}}\right]+\frac{\alpha}{2 H(1)}\left\{\frac{9}{t^{2}}-5 F(1)\right\} \\
=\sum_{k=1}^{\infty} \frac{2(2 k+1)^{2}}{H(k)(k+1)}\left\{k(k+1) F(k)-\frac{1}{t^{2}} \frac{1}{4(4 k+3)} \frac{1}{t^{2 k-2}}\right. \\
+\sum_{k=2}^{\infty} \frac{F(k)}{H(k)}\left\{(2 k+1)(2 k-1) \frac{1}{t^{2}}+F(k) \frac{4 k+1}{4 k-1}\right\} \\
\times\left\{\frac{1}{(r t)^{2 k-2} r^{2}}\left(\frac{\left(\frac{1}{2}\right)_{k}}{k !}\right)\right. \\
\left.+\frac{H(k+3) 2 k G(k)-1)\}}{1}\right\}
\end{gathered}
$$

\footnotetext{
0@IJTSRD—Available Online@www.ijtsrd.com-Volume-2-Issue-3-Mar-Apr 2018 Page:988
} 
$\times 2 k\left(\frac{\left(\frac{1}{2}\right)_{k}}{k !}\right)^{2} \frac{1}{(r t)^{2 k-2}}+\frac{\alpha}{2 H(1)}\left\{-5 F(1)+\frac{9}{t^{2}}\right\}$.

Using

$$
\tilde{A}_{1} f_{1}\left(\frac{1}{2}, 0\right)+\tilde{A}_{2} f_{3}\left(\frac{1}{2}, 0\right)=\tilde{A}_{1}-\tilde{A}_{2} \frac{1}{2 t^{2}}
$$

we finally obtain

$$
\begin{gathered}
-\frac{2}{\pi} S(r, t)=\frac{1+\nu}{3} \frac{1}{r^{3} t^{2}}+\sum_{k=1}^{\infty} \frac{2}{H(k)}\left[\frac{2(2 k+1)^{2}}{(k+1) r^{2}}\right. \\
\times\left\{k(k+1) F(k)-\frac{1}{t^{2}} \frac{1}{4(4 k+3)}\{(2 k-1)(4 k+3)\right. \\
\times 2 k G(k)-H(k) G(k-1)\}\}+k F(k)\{(2 k+2) \\
\left.\left.\times(2 k-1) \frac{1}{t^{2}}+F(k) \frac{4 k+1}{4 k-1}\right\}\right] \\
\times\left(\frac{(2 k-1) ! !}{(2 k) ! !}\right)^{2} \frac{1}{(r t)^{2 k} r} .
\end{gathered}
$$

The above equation completely agrees with the where result by Atsumi and Shindo.

A quantity of physical interest is the stress intensity factor which is given as

$$
K=\lim _{r \rightarrow \gamma^{+}} \sqrt{2(r-\gamma)} \sigma_{z z}(r, 0) .
$$

We choose $p(r)=p_{0}$ and put $\gamma-1=a$. We let

$$
r=\frac{a}{2}(s+1)+1, \quad t=\frac{a}{2}(\tau+1)+1,
$$

and in order to facilitate numerical analysis, assume $g(t)$ to have the following form:

$$
g(t)=p_{0}(t-1)^{\frac{1}{2}}(\gamma-t)^{-\frac{1}{2}} \tilde{G}(t) .
$$

With the aid of (3.24), $g(\tau)$ can be rewritten as

$$
g(\tau)=p_{0} \tilde{G}(\tau)\left(\frac{1+\tau}{1-\tau}\right)^{\frac{1}{2}} .
$$

The stress intensity factors $K$ can therefore be expressed in terms of $\tilde{G}(t)$ as

$$
K / p_{0}=\sqrt{2 a} \tilde{G}(\gamma),
$$

or in terms of the quantity actually calculated

$$
K / p_{0} \sqrt{a}=\sqrt{2} \tilde{G}(\gamma) .
$$

\section{Numerical analysis.}

In order to obtain numerical solution of (3.23), substitutions are made by the application of
(3.24) and (3.26) to obtain the following expression:

$$
\begin{gathered}
\frac{a}{\pi} \int_{-1}^{1}\left(\frac{1+\tau}{1-\tau}\right)^{\frac{1}{2}} \tilde{G}(\tau)\left[\frac{a}{2}(\tau+1)+1\right][R(s, \tau) \\
+S(s, \tau)] d \tau=1, \quad-1<s<1
\end{gathered}
$$

The numerical solution technique is based on the collocation scheme for the solution of singular integral equations given by Erdogan, Gupta, and Cook [11]. ${ }^{0}$ This amounts to applying a Gaussian quadrature formula for approximating the integral of a function $f(\tau)$ with weight function $[(1+\tau) /(1-\tau)]^{\frac{1}{2}}$ on the interval $[-1,1]$. Thus, letting $n$ be the number of quadrature points,

$$
\int_{-1}^{1}\left(\frac{1+\tau}{1-\tau}\right)^{\frac{1}{2}} f(\tau) d \tau \doteqdot \frac{2 \pi}{2 n+1} \sum_{k=1}^{n}\left(1+\tau_{k}\right) f\left(\tau_{k}\right),
$$

$$
\tau_{k}=\cos \left(\frac{2 k-1}{2 n+1}\right) \pi, \quad k=1, \ldots n .
$$

The solution of the integral equation is obtained by choosing the collocation points:

$$
s_{i}=\cos \left(\frac{2 i \pi}{2 n+1}\right), \quad i=1, \ldots, n,
$$

and solving the matrix system for $G^{*}\left(\tau_{k}\right)$ :

$$
\begin{gathered}
\sum_{k=1}^{n}\left[R\left(s_{j}, \tau_{k}\right)+S\left(s_{j}, \tau_{k}\right)\right] G^{*}\left(\tau_{k}\right)=\frac{2 n+1}{2 a}, \\
j=1, \ldots, n
\end{gathered}
$$

where

$$
\tilde{G}\left(\tau_{k}\right)=\frac{G^{*}\left(\tau_{k}\right)}{\left(1+\tau_{k}\right)\left[\frac{a}{2}\left(\tau_{k}+1\right)+1\right]} .
$$

\section{Numerical results and consideration.}

Numerical calculations have been carried out for $\nu=0.3$. The values of normalized stress intensity factor $K / p_{0} \sqrt{a}$ versus $a$ are shown in Fig.1-3 for various values of $\delta$.

Fig. 1 shows the variation of $K / p_{0} \sqrt{a}$ with respect to $a$ when $\delta=0$.

This figure shows that as $a$ increases, S.I.F. decreases steadily.

\footnotetext{
0@IJTSRD—Available Online@www.ijtsrd.com-Volume-2-Issue-3-Mar-Apr 2018 Page:989
} 
Fig. 2 and 3 deal with the cases when $\delta=0.3$ the overflow, computations could not be accomand $\delta=0.5$, respectively. Here we omit units. plished beyond the values $\delta>0.5$. And we found We can see that the trend is similar. Theoreti- that the variation of SIF is very small with recally the infinite series involved converges when spect to the variation of $\delta$. $\delta<1$ by comparison test. However, because of

0
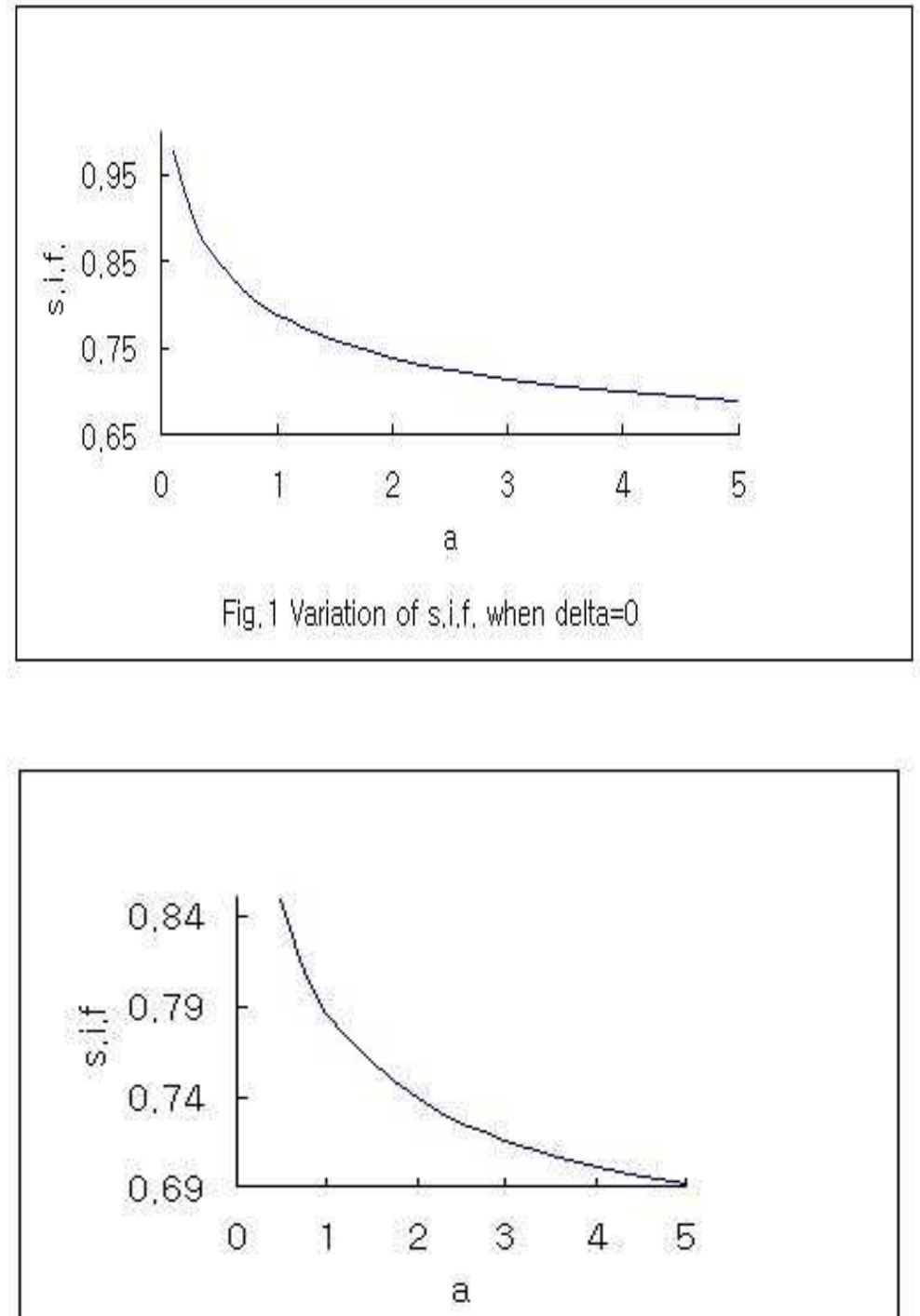

Fig. 2, variation of s.i.f, when delta $=, 1$

\footnotetext{
0@IJTSRD— Available Online@www.ijtsrd.com—Volume-2-Issue-3-Mar-Apr 2018 Page:990
} 


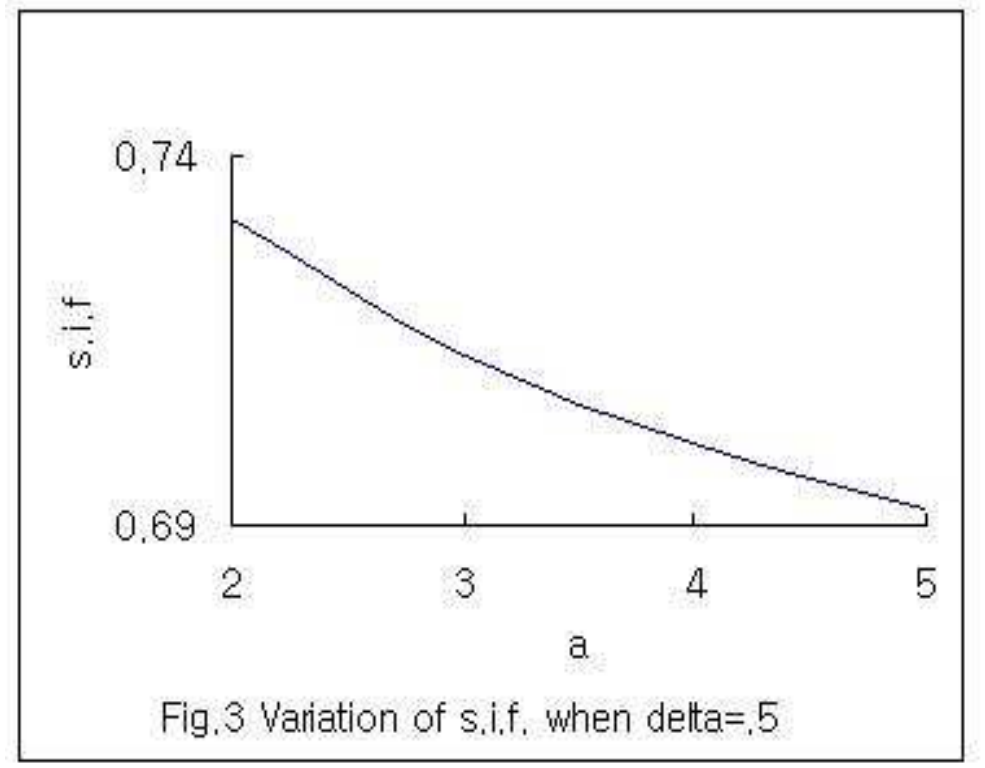

\section{Penny-shaped crack.}

In this section we are concerned with a pennyshaped crack in a convex lens shaped elastic material. The problem of determining the distribution of stress in an elastic sphere containing a penny-shaped crack or the mixed boundary value problems concerning a spherical boundary has been investigated by several researchers. Srivastava and Dwivedi [12] considered the problem of a penny-shaped crack in an elastic sphere, whereas Dhaliwal et al.[13] solved the problem of a penny-shaped crack in a sphere embedded in an infinite medium. On the other hand, Srivastav and Narain [14] investigated the mixed boundary value problem of torsion of a hemisphere.

7.Formulation of problem and reduction to a Fredholm integral equation of the second kind. ${ }^{0}$ We employ cylindrical coordinates $(r, \phi, z)$ with the plane $z=0$ coinciding the plane of the penny shaped crack. The center of the crack is located at $(\mathrm{r}, \mathrm{z})=(0,0)$. As before, spherical coordinates $(\rho, \theta, \phi)$ are connected with the cylindrical coordinates by

$$
z=\rho \cos \theta, \quad r=\rho \sin \theta
$$

Spherical coordinates $(\zeta, \vartheta, \phi)$ whose origin is at $z=-\delta, \quad r=0$, and is the center of the upper spherical surface of the convex elastic body, is also used. The elastic body is symmetrical with respect to the plane $z=0$.

The crack occupies the region $z=0,0 \leq$ $r \leq 1$. The radius of the spherical portion is $\zeta_{0}=\sqrt{\gamma^{2}+\delta^{2}}$. The boundary conditions are:

On the plane $z=0$, we want the continuity of the shear stress, and the normal displacement:

$$
\begin{array}{cc}
u_{z}\left(r, 0^{+}\right)-u_{z}\left(r, 0^{-}\right)=0, & 1 \leq r \leq \gamma, \\
\sigma_{r z}\left(r, 0^{+}\right)-\sigma_{r z}\left(r, 0^{-}\right)=0, & 0 \leq r \leq \gamma .
\end{array}
$$

And the crack is subjected to a known pressure $p(r)$, i.e.,

$$
\sigma_{z z}\left(r, 0^{+}\right)=-p(r), \quad 0 \leq r \leq 1 .
$$

On the surface of the spherical portion, stresses are zero:

$$
\begin{aligned}
& \sigma_{\zeta \zeta}\left(\zeta_{0}, \vartheta\right)=0 \\
& \sigma_{\zeta \vartheta}\left(\zeta_{0}, \vartheta\right)=0 .
\end{aligned}
$$

We can make use of (2.6)-(2.9) also, for the present case. The functions $\phi^{(1)}$ and $\phi^{(2)}$ for the regions $z>0$ and $z<0$, respectively, are chosen as follows:

$$
\phi^{(1)}(r, z)=(2 \nu-1) \int_{0}^{\infty} \xi^{-1} A(\xi) J_{0}(\xi r) e^{-\xi z} d \xi
$$

\footnotetext{
0@IJTSRD—Available Online@www.ijtsrd.com—Volume-2-Issue-3-Mar-Apr 2018 Page:991
} 


$$
\begin{gathered}
+\sum_{n=0}^{\infty} a_{n} \rho^{n} P_{n}(\cos \theta), \\
\phi^{(2)}(r, z)=(2 \nu-1) \int_{0}^{\infty} \xi^{-1} A(\xi) J_{0}(\xi r) e^{\xi z} d \xi \\
-\sum_{n=0}^{\infty} a_{n}(-1)^{n} \rho^{n} P_{n}(\cos \theta) .
\end{gathered}
$$

Here the superscripts (1) and (2) are taken for the region $z>0$ and $z<0$, respectively. The functions $\psi^{(1)}$ and $\psi^{(2)}$ are chosen as follows:

$$
\begin{gathered}
\psi^{(1)}(r, z)=\int_{0}^{\infty} A(\xi) J_{0}(\xi r) e^{-\xi z} d \xi \\
+\sum_{n=0}^{\infty} b_{n} \rho^{n} P_{n}(\cos \theta), \\
\psi^{(2)}(r, z)=-\int_{0}^{\infty} A(\xi) J_{0}(\xi r) e^{\xi z} d \xi \\
+\sum_{n=0}^{\infty} b_{n}(-1)^{n} \rho^{n} P_{n}(\cos \theta) .
\end{gathered}
$$

Then we can immediately satisfy condition (7.2) by these choice of functions (7.6)-(7.9).

Now the condition (7.1) requires

$$
\int_{0}^{\infty} A(\xi) J_{0}(\xi r) d \xi=0, \quad r>1
$$

Equation (7.10) is automatically satisfied by setting

$$
A(\xi)=\int_{0}^{1} g(t) \sin (\xi t) d t, g(0)=0 .
$$

Then from the boundary condition (7.3), we obtain

$$
\begin{gathered}
\int_{0}^{\infty} \xi A(\xi) J_{0}(\xi r) d \xi-\sum_{n=0}^{\infty} a_{2 n}(2 n)^{2} P_{2 n}(0) r^{2 n-2} \\
-2(1-\nu) \sum_{n=0}^{\infty} b_{2 n+1} P_{2 n+1}^{\prime}(0) r^{2 n}=-p(r) \\
0 \leq r \leq 1
\end{gathered}
$$

where the prime indicates the differentiation with respect to the argument. ${ }^{0}$
By substituting (7.11) into (7.12), it reduces to

$$
\begin{array}{r}
g(t)-\frac{2}{\pi} \sum_{n=0}^{\infty}(-1)^{n}\left\{2 n t^{2 n-1} a_{2 n}\right. \\
\left.+\alpha b_{2 n+1} t^{2 n+1}\right\}=h(t), \quad 0 \leq r \leq 1,
\end{array}
$$

where

$$
h(t)=-\frac{2}{\pi} \int_{0}^{t} \frac{r p(r)}{\sqrt{t^{2}-r^{2}}} d r .
$$

The solution will be complete, if the conditions on the surface of the spherical portion are satisfied.

\section{Conditions on the surface of the sphere.}

Equation (7.13) gives one relation connecting unknown coefficients $a_{n}$ and $b_{n}$. The stress components besides (2.8) and (2.9) which are needed for the present analysis are given by (3.1) and (3.2). To satisfy boundary conditions on the spherical surface, it is needed to represent $\phi, \psi$ in (7.6)-(7.9) in terms of $\zeta, \vartheta$ variables. To do so we utilize the following formula in the Appendix 2. An expression useful for the present analysis is the following

$$
P_{n}(\cos \theta) \rho^{n}=\sum_{k=0}^{n}\left(\begin{array}{l}
n \\
k
\end{array}\right) P_{k}(\cos \vartheta) \zeta^{k}(-\delta)^{n-k} .
$$

Thus

$$
\begin{aligned}
\sum_{n=0}^{\infty} a_{n} P_{n}(\cos \theta) \rho^{n} & =\sum_{n=0}^{\infty} a_{n} \sum_{k=0}^{\infty}\left(\begin{array}{c}
n \\
k
\end{array}\right) P_{k}(\cos \vartheta) \\
\times \zeta^{k}(-\delta)^{n-k} & =\sum_{j=0}^{\infty} P_{j}(\cos \vartheta) \zeta^{j} A_{j},
\end{aligned}
$$

where

$$
A_{j}=\sum_{n=j}^{\infty} \frac{n !}{(n-j) ! j !} a_{n}(-\delta)^{n-j} .
$$

Also

$$
-\frac{\partial}{\partial t} \int_{0}^{\infty} \xi^{-1} A(\xi) J_{0}(\xi r) e^{-\xi z} d \xi
$$

$$
=-\int_{0}^{1} g(t) \int_{0}^{\infty} J_{0}(\xi r) \cos (\xi t) e^{-\xi z} d \xi d t
$$

\footnotetext{
0@IJTSRD— Available Online@www.ijtsrd.com—Volume-2-Issue-3-Mar-Apr 2018 Page:992
} 
The inner integral of the above equation is

$$
\begin{gathered}
\int_{0}^{\infty} J_{0}(\xi r) \cos (\xi t) e^{-\xi z} d \xi \\
=\Re \int_{0}^{\infty} J_{0}(\xi r) e^{-\xi(z+i t)} d \xi \\
=\Re \frac{1}{\sqrt{r^{2}+(z+i t)^{2}}} \\
=\Re \frac{1}{\sqrt{r^{2}+z^{2}+2 z i t-t^{2}}} \\
=\Re \frac{1}{\rho \sqrt{1-2 \cos \theta\left(\frac{-i t}{\rho}\right)+\left(\frac{-i t}{\rho}\right)^{2}}} \\
=\frac{1}{\rho} \sum_{n=0}^{\infty}\left(\frac{t}{\rho}\right)^{2 n}(-1)^{n} P_{2 n}(\cos \theta) .
\end{gathered}
$$

Thus finally, from (8.2), and using the formula in Appendix, $\phi^{(1)}$ can be written in terms of spherical coordinates $(\zeta, \vartheta)$ as

$$
\phi^{(1)}=\sum_{k=0}^{\infty} P_{k}(\cos \vartheta)\left[\frac{\Phi_{k}}{\zeta^{k+1}}+A_{k} \zeta^{k}\right],
$$

where

$$
\begin{aligned}
\Phi_{k}=- & (\alpha-1) \sum_{n=0}^{[k / 2]} \frac{k !}{(2 n) !(k-2 n) !} \delta^{k-2 n} \\
& \times(-1)^{n} \int_{0}^{1} \frac{g(t) t^{2 n+1}}{2 n+1} d t .
\end{aligned}
$$

It is also necessary to express $\psi^{(1)}$ in terms of spherical coordinates $(\zeta, \vartheta)$. Now, as in $(8.2)$

$$
\sum_{n=0}^{\infty} b_{n} P_{n}(\cos \theta) \rho^{n}=\sum_{j=0}^{\infty} P_{j}(\cos \vartheta) \zeta^{j} B_{j},
$$

where

$$
B_{j}=\sum_{n=j}^{\infty} \frac{n !}{j !(n-j) !} b_{n}(-\delta)^{n-j} .
$$

${ }^{0}$ We first express following integral in $(\rho, \theta)$ coordinates

$$
\begin{aligned}
& \int_{0}^{\infty} A(\xi) J_{0}(\xi r) e^{-\xi z} d \xi=\int_{0}^{1} g(t) d t \\
& \times \int_{0}^{\infty} \sin (\xi t) J_{0}(\xi r) e^{-\xi z} d \xi .
\end{aligned}
$$

The inner integral on the right-hand side of (8.10) is

$$
\begin{array}{r}
-\Im \int_{0}^{\infty} J_{0}(\xi r) e^{-\xi(z+i t)} d \xi \\
=\sum_{n=0}^{\infty} \frac{P_{2 n+1}(\cos \theta)}{\rho^{2 n+2}}(-1)^{n} t^{2 n+1} .
\end{array}
$$

To express $(8.11)$ in the $(\zeta, \vartheta)$ spherical coordinates, we use the following formula in the Appendix 1.

$$
\begin{aligned}
& \frac{P_{2 n+1}(\cos \theta)}{\rho^{2 n+1}}=\sum_{k=0}^{\infty} \frac{(2 n+1+k) !}{k !(2 n+1) !} \\
& \times \delta^{k} \frac{P_{2 n+1+k}(\cos \vartheta)}{\zeta^{2 n+2+k}} .
\end{aligned}
$$

If we use (8.8) and (8.12), we get $\psi^{(1)}$ in spherical coordinates $(\zeta, \vartheta)$ as

$$
\psi^{(1)}=\sum_{k=0}^{\infty} P_{k}(\cos \vartheta)\left(\frac{\Psi_{k}}{\zeta^{k+1}}+B_{k} \zeta^{k}\right)+B_{0},
$$

where

$$
\begin{gathered}
\Psi_{k}=\sum_{n=0}^{[(k-1) / 2]} \frac{k !}{(2 n+1) !(k-2 n-1) !} \delta^{k-2 n-1} \\
\times(-1)^{n} \int_{0}^{1} g(t) t^{2 n+1} d t .
\end{gathered}
$$

Then if we substitute these values of $\phi^{(1)}$ and $\psi^{(1)}$ given by (8.6) and (8.13) into (3.2), and simplify the results by using the properties of Legendre polynomials, from the condition that on the spherical surface $\zeta=\zeta_{0}, \sigma_{\zeta \vartheta}=0$, we obtain the following equation

$$
\begin{gathered}
A_{k+1} k \zeta_{0}^{k-1}-B_{k+2} \zeta_{0}^{k+1} \frac{\alpha-(k+2)^{2}}{2 k+5} \\
-B_{k} \zeta_{0}^{k-1} \frac{k(2 \alpha-k-1)}{2 k+1}-\frac{k+3}{\zeta_{0}^{k+4}} \Phi_{k+1} \\
-\frac{\Psi_{k+2}}{\zeta_{0}^{k+4}} \frac{(k+3)(k+2+2 \alpha)}{2 k+5}+\frac{\Psi_{k}}{\zeta_{0}^{k+2}} \frac{\alpha-(k+1)^{2}}{2 k+1} \\
=0 .
\end{gathered}
$$

Likewise, from the condition $\sigma_{\zeta \zeta}\left(\zeta_{0}, \vartheta\right)=0$, we get the following equation,

$$
-A_{k+1} k(k+1) \zeta_{0}^{k-1}
$$

\footnotetext{
0@IJTSRD—Available Online@www.ijtsrd.com—Volume-2-Issue-3-Mar-Apr 2018 Page:993
} 
INTERNATIONAL JOURNAL OF TREND IN SCIENTIFIC RESEARCH AND DEVELOPMENT(IJTSRD)ISSN:2456-64T0

$$
\begin{gathered}
+B_{k+2} \zeta_{0}^{k+1} \frac{(k+2)\{2-\alpha-(k-1)(k+2)\}}{2 k+5} \\
+B_{k} \zeta_{0}^{k-1} \frac{k(k+1)(2 \alpha-k-1)}{(2 k+1)} \\
-\frac{(k+2)(k+3)}{\zeta_{0}^{k+4}} \Phi_{k+1} \\
-\Psi_{k+2} \frac{(k+2)(k+3)(k+2+2 \alpha)}{\zeta_{0}^{k+4}(2 k+5)} \\
-\Psi_{k} \frac{(k+1)\{(k+4)(k+1)+\alpha-2\}}{\zeta_{0}^{k+2}(2 k+1)} \\
=0 .
\end{gathered}
$$

Thus if we multiply (8.15) by $k+1$ and add (8.16), from the resulting equation we find

$$
\begin{gathered}
B_{k+2}=\frac{(2 k+5)}{\zeta_{0}^{k+1}\{-\alpha(2 k+3)+2(k+2)(k+3)\}} \\
\times\left[\frac{(k+3)(2 k+3)}{\zeta_{0}^{k+4}} \Phi_{k+1}\right. \\
+\Psi_{k+2} \frac{(2 k+3)(k+3)(k+2+2 \alpha)}{\zeta_{0}^{k+4}(2 k+5)} \\
\left.+\frac{\Psi_{k}}{\zeta_{0}^{k+2}}(k+1)(k+3)\right] .
\end{gathered}
$$

${ }^{0}$ Using the relation

$$
\Phi_{k+1}=-\frac{\alpha-1}{k+2} \Psi_{k+2},
$$

$B_{k+2}$ is

$$
\begin{gathered}
B_{k+2}=\frac{(2 k+5)}{\zeta_{0}^{k+1}\{-\alpha(2 k+3)+2(k+2)(k+3)\}} \\
\times\left[\Psi_{k+2} \frac{(2 k+3)(k+3)\left((k+3)^{2}-\alpha\right)}{\zeta_{0}^{k+4}(2 k+5)(k+2)}\right. \\
\left.+\frac{\Psi_{k}}{\zeta_{0}^{k+2}}(k+1)(k+3)\right] .
\end{gathered}
$$

From (8.15) we have

$$
A_{k+3}=\frac{\Psi_{k}}{\zeta_{0}^{2 k+3}} L(k)+\frac{\Psi_{k+2}}{\zeta_{0}^{2 k+5}} M(k)+\frac{\Psi_{k+4}}{\zeta_{0}^{2 k+7}} N(k),
$$

where

$$
\begin{gathered}
L(k)=(k+1)(k+3) \frac{(2 \alpha-k-3)}{\mathcal{H}(k)}, \\
M(k)=\frac{1}{k+2}\left[(k+3) \frac{(2 k+3)(2 \alpha-k-3) I(k)}{(2 k+5) \mathcal{H}(k)}\right.
\end{gathered}
$$

$$
\begin{gathered}
\left.+(k+4)(k+5) \frac{G(k)}{\mathcal{H}(k+2)}+\frac{G(k+2)}{2 k+5}\right], \\
N(k)=\left[\frac{G(k)}{\mathcal{H}(k+2)}(2 k+7)-1\right] \\
\times \frac{(k+5) I(k+2)}{(2 k+9)(k+2)(k+3)},
\end{gathered}
$$

with

$$
\mathcal{H}(k)=-\alpha(2 k+3)+2(k+2)(k+3),
$$

$G(k)=2-\alpha-(k+1)(k+4), \quad I(k)=-\alpha+(k+3)^{2}$.

Therefore using the formula in Theorem B of Appendix 4 , we have

$$
\begin{gathered}
A=\sum_{n=1}^{\infty}(-1)^{n} 2 n t^{2 n-1} a_{2 n} \\
=\sum_{n=1}^{\infty}(-1)^{n} 2 n t^{2 n-1} \frac{1}{\delta^{2 n}(2 n) !} \sum_{k=2 n}^{\infty} \frac{k ! A_{k} \delta^{k}}{(k-2 n) !} \\
=\sum_{k=2}^{\infty}\left[\sum_{n=1}^{[k / 2]} \frac{k !(-1)^{n}}{(2 n-1) !(k-2 n) !}\left(\frac{t}{\delta}\right)^{2 n} \frac{1}{t}\right] \\
\times A_{k} \delta^{k}=\sum_{k=-1}^{\infty} f_{k}(t) A_{k+3}=\sum_{k=-1}^{\infty} f_{k}(t) \\
\times\left[\frac{\Psi_{k}}{\zeta_{0}^{2 k+3}} L(k)+\frac{\Psi_{k+2}}{\zeta_{0}^{2 k+5}} M(k)+\frac{\Psi_{k+4}}{\zeta_{0}^{2 k+7}} N(k)\right] .
\end{gathered}
$$

In the above equation $\Psi_{k}=0$, if $k \leq 0$ and

$$
\begin{aligned}
f_{k}(t)=\delta^{k+3} \sum_{n=1}^{[(k+3) / 2]} \frac{(k+3) !(-1)^{n}}{(2 n-1) !(k+3-2 n) !} \\
\quad \times\left(\frac{t}{\delta}\right)^{2 n} \frac{1}{t} .
\end{aligned}
$$

If we substitute the values of $\Psi_{k}$ into (8.19),it reduces to

$$
A=\int_{0}^{1} g(u) \Omega_{1}(t, u) d u
$$

where

$$
\begin{gathered}
\Omega_{1}(t, u)=\sum_{k=-1}^{\infty} f_{k}(t)\left[\frac{h_{k}(u)}{\zeta_{0}^{2 k+3}} L(k)\right. \\
\left.+\frac{h_{k+2}(u)}{\zeta_{0}^{2 k+5}} M(k)+\frac{h_{k+4}(u)}{\zeta_{0}^{2 k+7}} N(k)\right] .
\end{gathered}
$$

\footnotetext{
0@IJTSRD—Available Online@www.ijtsrd.com-Volume-2-Issue-3-Mar-Apr 2018 Page:994
} 
IXTERNATIONAL JOURNAL OF TREND IN SCIENTIFIC RESEARCH AND DEVELOPMENT(IJTSRD)ISSN:2456-6470

In the above equation $h_{k}(u)=0$, if $k \leq 0$ and

$$
\begin{aligned}
h_{k}(u)=\delta^{k} \sum_{n=0}^{[(k-1) / 2]} \frac{(-1)^{n} k !}{(2 n+1) !(k-2 n-1) !} \\
\quad \times\left(\frac{u}{\delta}\right)^{2 n+1} \cdot
\end{aligned}
$$

$$
\begin{gathered}
=\zeta^{2}\left\{1-2 \cos \vartheta \frac{\delta}{\zeta}+\left(\frac{\delta}{\zeta}\right)^{2}\right\} \\
\frac{1}{\rho}=\frac{1}{\zeta \sqrt{1-2 \cos \vartheta \frac{\delta}{\zeta}+\left(\frac{\delta}{\zeta}\right)^{2}}}=\sum_{n=0}^{\infty} P_{n}(\cos \vartheta) \frac{\delta^{n}}{\zeta^{n+1}} \\
\frac{P_{n}(\cos \theta)}{\rho^{n+1}}=\frac{(-1)^{n}}{n !} \frac{\partial^{n}}{\partial z^{n}}\left(\frac{1}{\rho}\right) \\
=\sum_{k=0}^{\infty} \frac{(-1)^{n}}{n !} \frac{\partial^{n}}{\partial z^{n}}\left(\frac{P_{k}(\cos \vartheta)}{\zeta^{k+1}}\right) \delta^{k} \\
=\sum_{k=0}^{\infty} \frac{(-1)^{n}}{n !} \frac{\partial^{n+k}}{\partial z^{n+k}}\left(\frac{1}{\zeta}\right) \frac{(-1)^{k}}{k !} \delta^{k} \\
=\sum_{k=0}^{\infty}\left(\begin{array}{c}
n+k \\
k
\end{array}\right) \frac{P_{n+k}(\cos \vartheta)}{\zeta^{n+k+1}} \delta^{k} .
\end{gathered}
$$

Also, using Theorem B of Appendix 4, we get

$$
\begin{gathered}
\sum_{n=0}^{\infty}(-1)^{n} b_{2 n+1} t^{2 n+1}=\sum_{n=0}^{\infty}(-1)^{n} \frac{t^{2 n+1}}{(2 n+1) ! \delta^{2 n+1}} \\
\times \sum_{k=2 n+1}^{\infty} \frac{k ! B_{k} \delta^{k}}{(k-2 n-1) !} \\
=\sum_{k=1}^{\infty}\left[\sum_{n=0}^{\left[\frac{k-1}{2}\right]} \frac{(-1)^{n} k !}{(2 n+1) !(k-2 n-1) !}\left(\frac{t}{\delta}\right)^{2 n+1}\right] B_{k} \delta^{k}
\end{gathered}
$$$$
=\sum_{k=-1}^{\infty} h_{k+2}(t) B_{k+2}
$$

Appendix 2. Proof of (3.16).

$$
\begin{gathered}
=\sum_{k=-1}^{\infty} h_{k+2}(t) \frac{2 k+5}{\zeta_{0}^{k+1} \mathcal{H}(k)}\left[\frac{\Psi_{k}}{\zeta_{0}^{k+2}}(k+1)(k+3)\right. \\
\left.+\frac{\Psi_{k+2}}{\zeta_{0}^{k+4}} \frac{(k+3)(2 k+3)}{2 k+5} I(k)\right]
\end{gathered}
$$$$
=\int_{0}^{1} g(u) \Omega_{2}(t, u) d u,
$$

$$
\begin{gathered}
2 \pi \rho^{n} P_{n}(\cos \theta)=\int_{-\pi}^{\pi}(z+i x \cos u+i y \sin u)^{n} d u \\
=\int_{-\pi}^{\pi}(-\delta+Z+i x \cos u+i y \sin u)^{n} d u \\
=\sum_{k=0}^{n} \frac{n !}{(n-k) ! k !}(-\delta)^{n-k} \\
\times \int_{-\pi}^{\pi}(Z+i x \cos u+i y \sin u)^{k} d u \\
=2 \pi \sum_{k=0}^{n} \frac{n !}{(n-k) ! k !}(-\delta)^{n-k} \zeta^{k} P_{k}(\vartheta) .
\end{gathered}
$$

${ }^{0}$ where

$$
\begin{gathered}
\Omega_{2}(t, u)=\sum_{k=-1}^{\infty} h_{k+2}(t) \frac{2 k+5}{\zeta_{0}^{k+1} \mathcal{H}(k)}\left[\frac{h_{k}(u)}{\zeta_{0}^{k+2}}(k+1)\right. \\
\left.\times(k+3)+\frac{h_{k+2}(u)}{\zeta_{0}^{k+4}} \frac{(k+3)(2 k+3)}{2 k+5} I(k)\right] .
\end{gathered}
$$

In the above equation $h_{k}(u)=0$, if $k \leq 0$. Thus (7.13) reduces to the following Fredholm integral equation of the second kind

$$
g(t)+\int_{0}^{1} g(u) K(t, u) d u=h(t)
$$

where

$$
K(t, u)=-\frac{2}{\pi}\left\{\Omega_{1}(t, u)+\alpha \Omega_{2}(t, u)\right\}
$$

Appendix 1. Proof of (3.3). Since

$$
\rho^{2}=(\zeta \cos \vartheta-\delta)^{2}+(\zeta \sin \vartheta)^{2}
$$

Appendix 3. Theorem A. If the equation

$$
B_{k}=\sum_{i=0}^{k} \frac{k !}{(k-i) ! i !} a_{i}
$$

is solved for $a_{i}^{\prime} s$, it will be written as

$$
a_{i}=\sum_{k=0}^{i} \frac{i !}{(i-k) ! k !} B_{k}(-1)^{i-k} .
$$

Proof. We prove it by mathematical induction. Suppose it is true for $i$, we will show that it is also true for $i+1$. Suppose $B_{i+1}$ is given by

$$
B_{i+1}=\sum_{k=0}^{i+1} \frac{(i+1) !}{(i+1-k) ! k !} a_{k}=a_{i+1}
$$

\footnotetext{
0@IJTSRD—Available Online@www.ijtsrd.com-Volume-2-Issue-3-Mar-Apr 2018 Page:995
} 


$$
+\sum_{k=0}^{i} \frac{(i+1) !}{(i+1-k) ! k !} a_{k}
$$

Thus

$$
\begin{gathered}
a_{i+1}=B_{i+1}-\sum_{k=0}^{i} \frac{(i+1) !}{(i+1-k) ! k !} \\
\times \sum_{j=0}^{k} B_{j} \frac{k !}{j !(k-j) !}(-1)^{k-j} \\
=B_{i+1}-\sum_{j=0}^{i} B_{j} \frac{(i+1) !}{j !} \sum_{k=j}^{i} \frac{(-1)^{k-j}}{(i+1-k) !(k-j) !} .
\end{gathered}
$$

The inner summation in the above equation can written as, by changing the variable $k-j=m$

$$
\begin{aligned}
& \sum_{k=j}^{i} \frac{(-1)^{k-j}}{(i+1-k) !(k-j) !} \\
= & \sum_{m=0}^{i-j} \frac{(-1)^{m}}{(i+1-m-j) ! m !} \\
= & -\frac{(-1)^{i-j+1}}{(i-j+1) !}
\end{aligned}
$$

${ }^{0}$ since

$$
\sum_{m=0}^{i-j+1} \frac{(-1)^{m}(i-j+1) !}{(i+1-m-j) ! m !}=(1-1)^{i-j+1}=0 .
$$

Then (A.1) is equal to

$$
\begin{aligned}
a_{i+1} & =B_{i+1}+\sum_{j=0}^{i} \frac{B_{i}(i+1) !(-1)^{i+1-j}}{j !(i+1-j) !} \\
& =\sum_{j=0}^{i+1} \frac{B_{i}(i+1) !(-1)^{i+1-j}}{j !(i+1-j) !} .
\end{aligned}
$$

Appendix 4. Theorem B. If the equation

$$
B_{k}=\sum_{n=k}^{\infty}\left(\begin{array}{c}
n \\
k
\end{array}\right)(-\delta)^{n-k} a_{n}
$$

is solved for $a_{n}^{\prime} s$, it will be written as

$$
a_{n}=\sum_{k=n}^{\infty}\left(\begin{array}{c}
k \\
n
\end{array}\right) \delta^{k-n} B_{k} .
$$

Proof. Let

$$
\sum_{n=0}^{\infty} a_{n}(-\delta)^{n} x^{n}=f(x)
$$

then

$$
a_{n}(-\delta)^{n} n !=f^{(n)}(0)
$$

and

$$
f^{(k)}(1)=\sum_{n=k}^{\infty} a_{n}(-\delta)^{n} \frac{n !}{(n-k) !}=k ! B_{k}(-\delta)^{k} .
$$

From Taylor's series

$$
f(x)=\sum_{k=0}^{\infty} \frac{f^{(k)}(1)}{k !}(x-1)^{k} .
$$

Thus

$$
\begin{aligned}
& a_{n}(-\delta)^{n} n !=f^{(n)}(0)=\left.\sum_{k=0}^{\infty} \frac{f^{(k)}(1)}{k !} \frac{d^{n}}{d x^{n}}(x-1)^{k}\right|_{x=0} \\
& =\sum_{k=n}^{\infty} \frac{f^{(k)}(1)}{(k-n) !}(-1)^{k-n}=\sum_{k=n}^{\infty} \frac{B_{k}(-1)^{k-n}(-\delta)^{k} k !}{(k-n) !} .
\end{aligned}
$$

Therefore

$$
a_{n}=\sum_{k=n}^{\infty} \frac{k !}{n !(k-n) !} B_{k} \delta^{k-n} .
$$

\section{References}

[1] L. M. Keer, V.K. Luk, and J. M. Freedman, Circumferential edge crack in a cylindrical cavity, J.Appl.Mech. 44, 1977, pp.250-254

[2] L. M. Keer and H. A. Watts, Mixed boundary value problems for a cylindrical shell, Int.J.Solids Structures, 12, 1976, pp.723-729

[3] A. Atsumi and Y. Shindo, Axisymmetric singular stresses in a thick-walled spherical shell with an internal edge crack, J.Appl.Mech. 50,1983, pp.37-42.

[4] A. Atsumi and Y. Shindo, Peripheral edge crack around spherical cavity under uniaxial tension field, Int. J. Engng Sci. 21(3), 1983, pp.269278

[5] D.-S. Lee, Torsion of a long circular cylinder having a spherical cavity with a peripheral edge crack, Eur.J.Mech.A/Solids 21, 2002, pp.961-969

\footnotetext{
0@IJTSRD—Available Online@www.ijtsrd.com—Volume-2-Issue-3-Mar-Apr 2018 Page:996
} 
[6] D.-S. Lee, Tension of a long circular cylin- [11] F. Erdogan, G.D. Gupta, and T.S. Cook, der having a spherical cavity with a peripheral Numerical solution of singular integral equation. edge crack, Int. J. Solids Structures, 40, 2003, Methods of analysis and solutions of crack probpp.2659-2671

[7] H. Wan, Q. Wang, and X. Zhang, Closed form solution of stress intensity factors for cracks emanating from surface semi-spherical cavity in finite body with energy release rate method, Appl.Math.Mech.Engl.Ed., 37(12), 2016, pp.1689-1706

[8] A.E. Green and W. Zerna, Theoretical Elasticity. New York, Oxford Univ. Press, 1975. lems, Noordhoff International Publishing, 1973, pp.368- 425

[12] K.N. Srivastava and J.P. Dwivedi, The effect of a penny-shaped crack on the distribution of stress in an elastic sphere, Int.J.Engng. sci., 9, 1971, pp.399-420,

[13] Ranjit S.Dhaliwal, Jon G. Rokne, and Brij M. Singh, Penny-shaped crack in a sphere embedded in an infinite medium, Int.J.Engng Sci.

[9] E.T. Whittaker and G.N. Watson, A Course of Modern Analysis, Cambridge Univ. Press, Cambridge, $1973 .^{0}$

17, 1979, pp.259-269

[14] R.P.Srivastav and Prem Narain, Some mixed boundary value problems for regions with spher[10] A. Erdélyi, W. Magnus, F. Oberhettinger, and F.G. Tricomi, Tables of Integral Transforms ical boundary, Journal of Math. Mech., 14, 1965, pp.613-627

Vol.I, New York, McGraw-Hill, 1954

0@IJTSRD— Available Online@www.ijtsrd.com-Volume-2-Issue-3-Mar-Apr 2018 Page:997 